\title{
BMJ Open Multicentre, single-blind randomised controlled trial comparing MyndMove neuromodulation therapy with conventional therapy in traumatic spinal cord injury: a protocol study
}

Kim D Anderson (D) , 1,2 James R Wilson, ${ }^{1,2}$ Radha Korupolu, ${ }^{3,4}$ Jacqueline Pierce, ${ }^{5}$ James M Bowen (1) , 6,7 Daria O'Reilly, ${ }^{6,8}$ Naaz Kapadia, 9,10 Milos R Popovic, ${ }^{11,12}$ Lehana Thabane, ${ }^{6,13}$ Kristin E Musselman ${ }^{9,10}$

To cite: Anderson KD, Wilson JR, Korupolu R, et al. Multicentre, singleblind randomised controlled trial comparing MyndMove neuromodulation therapy with conventional therapy in traumatic spinal cord injury: a protocol study. BMJ Open 2020;10:e039650. doi:10.1136/ bmjopen-2020-039650

- Prepublication history for this paper is available online. To view these files, please visit the journal online (http://dx.doi. org/10.1136/bmjopen-2020039650).

Received 21 April 2020 Revised 20 August 2020 Accepted 27 August 2020

Check for updates

(C) Author(s) (or their employer(s)) 2020. Re-use permitted under CC BY-NC. No commercial re-use. See rights and permissions. Published by BMJ.

For numbered affiliations see end of article.

Correspondence to Dr Kristin E Musselman; Kristin.Musselman@uhn.ca

\section{ABSTRACT}

Introduction This protocol is describing a multicentre, single-blind randomised controlled trial. The objective is to compare the efficacy of MyndMove therapy versus conventional therapy (CT) in improving upper extremity function in individuals with $\mathrm{C} 4-\mathrm{C} 7$ traumatic, incomplete spinal cord injury (SCI). It is being conducted in two US and two Canadian $\mathrm{SCl}$ rehabilitation centres.

Methods and analysis Sixty people aged 18 years or older with a C4-C7 incomplete (AIS B-D) SCI between 4 months and 8 years postinjury are randomised to receive 40 sessions of MyndMove neuromodulation therapy or CT within a 14-week period of time. Therapy sessions are 1 hour in duration with a dose of 3-5 sessions per week. Assessments occur prior to randomisation, after 20 sessions, after 40 sessions and 10 weeks after the last session. The primary outcome measure is the efficacy of MyndMove therapy versus CT in improving upper extremity function as measured by Spinal Cord Independence Measure III: Self-Care subscore after 40 sessions. Secondary outcomes include: (1) improvements in the SCIM mobility subscore; (2) upper limb functions measured by Graded Redefined Assessment of Strength, Sensibility and Prehension and (3) Toronto Rehab Institute Hand Function Test; (4) To assess safety as measured by serious and non-serious adverse events recorded for participants in both groups of the study population over the duration of the study; (5) to compare the change in quality of life as measured by the Spinal Cord Injury-Quality of Life; and (6) to evaluate the impact on healthcare resource utilisation. Ethics and dissemination All ethical approvals were obtained prior to enrolling any participants. Dissemination of the results of the study will be made at peer-reviewed academic meetings and through peerreviewed medical journals

Trial registration number NCT03439319

\section{INTRODUCTION}

Spinal cord injury (SCI) is a devastating, life-altering event that can lead to significant disability, in addition to socioeconomic

\section{Strengths and limitations of this study}

A strength of this study is that it is a properly powered randomised controlled trial designed to detect functionally meaningful change in participants with tetraplegia.

- This therapy requires the use of a device that is not currently part of standard rehabilitation for spinal cord injury and, as a result, the participant and treating therapist are not blinded.

- The assessing therapist is blinded to reduce the risk of bias.

The statistical analysis team is blinded to the study group.

challenges for the individual, family and community at large. A survey of people with SCI revealed that the majority of people with tetraplegia (which constitutes more than $50 \%$ of individuals with SCI) rated recovery of hand function as their highest priority. ${ }^{12}$ Currently, various approaches to improve hand function after SCI are used, for example: exercises, biofeedback, robotic therapy, task-specific movement therapy, reconstructive surgeries and functional electrical stimulation (FES) therapy. To date, FES therapy has been found to be one of the most promising approaches in improving voluntary hand function. ${ }^{3-18}$ One school of thought proposes that FES can be used as a short-term therapeutic intervention to help improve voluntary grasping function. A number of FES systems have been used for this application, for example: NESS H200 $0^{4-6}$; the Bionic Glove and its newer version HandEstim Wireless Hand Stimulator ${ }^{8}$; and the complex motion system. ${ }^{12} 18$ Emerging evidence in tetraplegia suggests that 
electrical stimulation and FES therapy improve arm and hand function more than conventional therapy (CT), particularly when provided in combination with various types of CT. Recently, a study demonstrated that the functional benefits of massed practice of CT were greater when augmented by sensory stimulation. ${ }^{19}$ Another study demonstrated that exercise therapy combined with FES produced greater functional improvements compared with exercise therapy combined with traditional electrical stimulation. ${ }^{20}$

MyndMove therapy is a non-invasive FES neuromodulation therapy designed to restore voluntary reaching and grasping movements in individuals paralysed by SCI or stroke. It is based on FES principles and therapeutic interventions $^{21}$ to provide clinically meaningful gains in both upper extremity function and self-care functional independence. ${ }^{22}$ The MyndMove system promotes development and re-establishment of neural pathways within the central nervous system (CNS) and between CNS and the upper extremities by engaging neuroplasticity following neurological injury. ${ }^{23}$ Therapists use the device with surface electrodes to deliver proprietary electrical stimulation sequences to induce targeted muscle contractions leading to functional movements. Over multiple sessions, the treatments are thought to reconnect the signal from the brain to the muscles, restoring voluntary use of their arms and hands. MyndMove therapy is approved for sale by Health Canada (licence number 93158) and has been confirmed by the FDA (510(k) Number K170564). MyndMove therapy has also been confirmed by FDA to be a non-significant risk device and exempt from an Investigational Device Exemption (IDE; reference file Q131135).

A pilot study comparing the effectiveness of FES neuromodulation therapy to CT has been conducted in individuals with cervical, incomplete SCI. ${ }^{24}$ In that study, a small number of participants with chronic C4-C7 American Spinal Injury Association Impairment Scale (AIS) B-D SCI were randomised to FES neuromodulation therapy or CT and received 39 hours of therapy over 13-16 weeks. The FES neuromodulation therapy group improved fivefold on the primary outcome measure (Toronto Rehabilitation Institute-Hand Function Test) compared with the CT group. However, because there were only eight people enrolled and the study was open label, a larger randomised controlled trial (RCT) with blinded assessments was needed to definitively compare the effectiveness of the two interventions.

The protocol for this multicentre RCT in people with tetraplegia following traumatic SCI aims to: (1) confirm the FES neuromodulation treatment effect as delivered by the MyndMove device across multiple investigational sites, (2) characterise the long-term benefits and retention of function by including long-term follow-up assessments and (3) compare the efficacy of MyndMove therapy to an equivalent number of hours of CT. The study will also evaluate the impact of MyndMove therapy on the quality of life for people with traumatic SCI (C4-C7) over the course of 24 weeks. Ultimately, the data from these

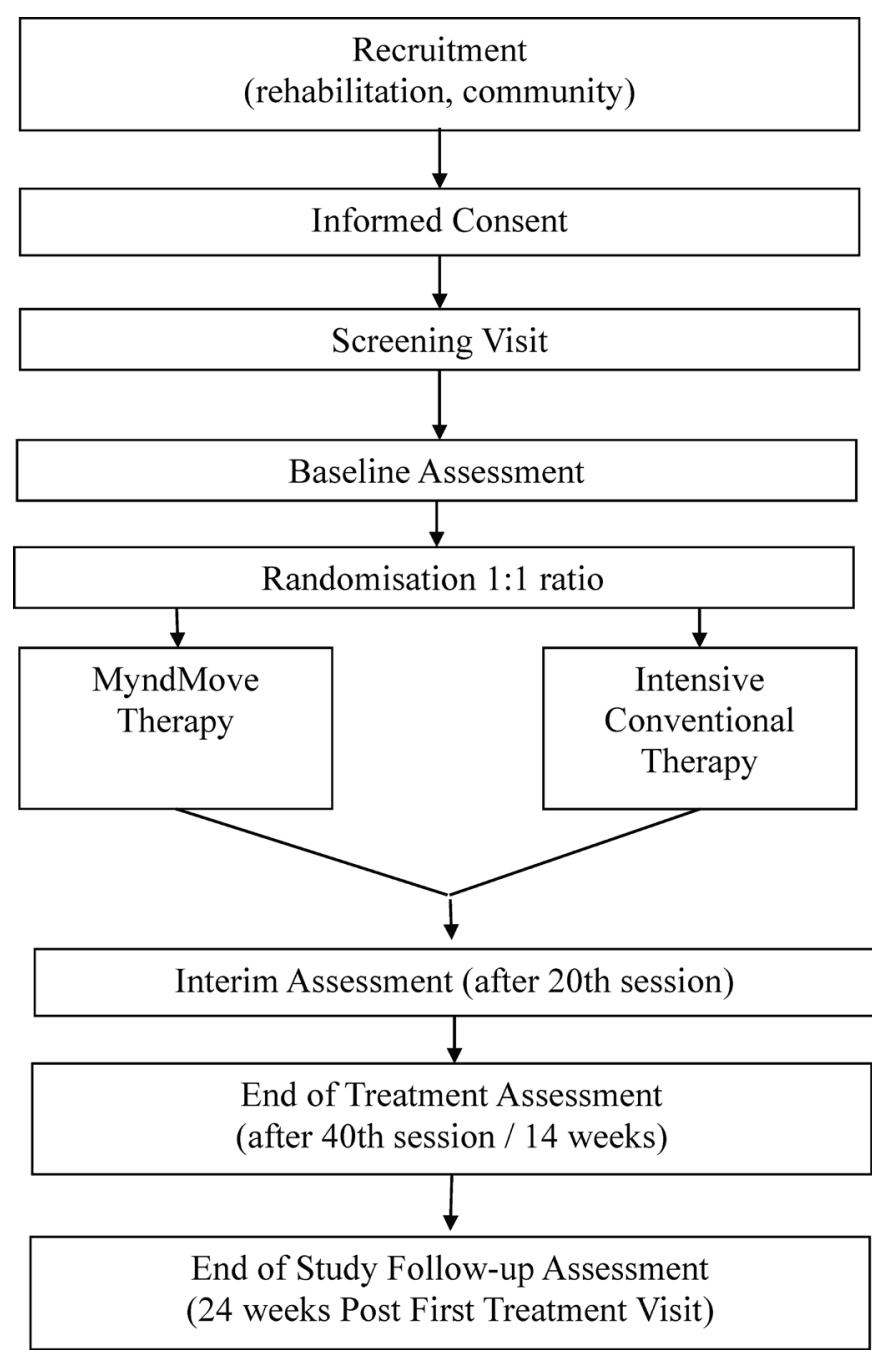

Figure 1 CONSORT diagram of study flow chart. CONSORT, Consolidated Standards of Reporting Trials.

studies will assist in redefining clinical best practices in SCI rehabilitation.

\section{METHODS AND ANALYSIS}

Trial design and setting

This study is designed as a multicentre, parallel group, twoarm, single-blind, RCT to compare the clinical outcomes of MyndMove therapy to CT for individuals with C4-C7 traumatic incomplete SCI with upper extremity paresis. See figure 1 for the study flow chart. The study is being conducted at four regional rehabilitation medical centres, in Canada and the USA, that specialise in providing neurorehabilitation to people with SCI. The first participant was enrolled in June 2019. It is estimated that the final participant will be enrolled by December 2020, but this may be negatively impacted by COVID-19.

\section{Recruitment and retention}

Each of the investigational sites has experience in recruiting individuals with SCI for clinical studies, and each investigational site has a study coordinator assigned to the study who routinely reviews charts to identify 
potential study participants and to increase awareness of the planned clinical study within their community. Recruitment strategies include outreach to advocacy and support groups for individuals with SCI. Study coordinators will enhance retention of participants by developing rapport with them during the active portion of the trial, then periodically communicating with participants during the follow-up portion of the trial.

\section{Eligibility criteria}

Inclusion criteria:

1. Traumatic incomplete (AIS B-D) C4-C7 SCI.

2. Paralysis or paresis in both upper extremities.

3. At least 4 months (120 days) and less than 96 months (2920 days) post-traumatic SCI.

4. Baseline Spinal Cord Independence Measure III: Self-Care (SCIM-SC) $\leq 10$.

5. From an inpatient (such as skilled nursing facility) or outpatient care setting.

6. Able to understand and follow instructions.

7. Able to tolerate being in a seated position for a least 1 hour required to deliver upper limb therapy.

8. Willing to attend treatment sessions and all assessment sessions.

9. Able to understand and provide informed consent.

10. Male and female participants $\geq 18$ years of age at the time of enrolment.

Exclusion criteria:

1. Previous history of any other neuromuscular disorder or conditions that may affect motor response.

2. Upper extremity injury or condition prior to SCI that limits the function of the hand or arm.

3. Malignant skin lesion on the affected upper extremity.

4. Rash or open wound at any potential electrode site.

5 . History of seizure disorder not effectively managed by seizure medications.

6. An implanted metallic part (eg, plates, screws or joint replacement) or electrical device (eg, implantable cardiac defibrillator, pacemaker and spinal stimulation). (Note: if the participant has passive metallic implants, the therapy can be delivered if the implants are located in an area other than where the electrical stimulant is to be delivered.)

7. Complete denervation of muscles that are targeted by MyndMove such that MyndMove is unable to elicit tetanic muscle contraction when upper limits of stimulation intensity (of the device) for the targeted muscle are applied.

8. Poorly controlled autonomic dysreflexia (as determined by the local site physician).

9. History of psychiatric illness requiring hospitalisation within past 24 months.

10. Active drug treatment for dementia.

11. Life expectancy of less than 12 months due to other illness.

12. In the judgement of the medical provider, participant has medical complications that may interfere with the execution of the study.
13. Currently enrolled in another upper limb intervention study and/or has received MyndMove therapy within the past 3 months.

14. Enrolled, in the past 6 months, in a clinical study involving drugs or biologicals.

15. Currently dependent on a ventilator.

16. Botulinum toxin injection into affected upper extremity and the muscle targeted by MyndMove therapy within 6 months prior to the study start. No botulinum toxin injections in the upper extremity during the study treatment and follow-up period.

17. Women who are pregnant or planning to become pregnant in the duration of the trial.

18. Regional disorder of the upper extremities such as fracture, dislocation or joint contractures to less than $50 \%$ of expected range of motion.

\section{Sample size}

The sample size calculation is based on the test of the research hypothesis that the mean difference in SCIM-SC in the MyndMove intervention group is better than CT control group. The primary measure of effect is the difference in function measured using SCIM-SC at 14 weeks. The criterion for significance (alpha) has been set at 0.05 . The test is two tailed, which implies that a mean difference in either direction will be interpreted. The sample was calculated using the power procedure in SAS V.9.2. With the proposed sample size of 30 in each of the two groups (ie, assuming a 1:1 allocation ratio) (ie, total sample size of 60 ), the study will have power of at least $80 \%$ to yield a statistically significant result using t-test (assuming an intention-to-treat principle for the analysis) of the difference between mean SCIM-SC scores at 14 weeks adjusting for baseline SCIM-SC scores at alpha $=0.05$. It is important to note that using the assumption of a t-test is more conservative in that an analysis of variance (ANCOVA) will lead to better power. This computation assumes that SCIM-SC scores are normally distributed, the mean difference is 3 points and the common within-group SD is 4.05 . These estimates are modified estimates from the pilot study, ${ }^{24}$ which account for the type of intervention planned for in this study. The assumed minimal clinically important difference is considered to correspond to a substantially meaningful improvement on the SCIM-SC, approximately 3 points, ${ }^{25}{ }^{26}$ and also represents a moderate effect of the intervention.

\section{Allocation and blinding}

Study participants will be stratified by rehabilitation site and will be allocated in a 1:1 ratio to the following two treatment arms:

1. MyndMove therapy: participants will receive a minimum-maximum of 36-40 1-hour sessions per day of MyndMove therapy within a 14-week period of time.

2. CT: participants will receive upper-limb CT of equivalent frequency, intensity and duration to MyndMove 
therapy (ie, a minimum-maximum of 36-40 1-hour sessions per day of CT within a 14-week period of time).

The randomisation schedule will be generated and maintained by a statistician at the Biostatistics Unit. A 1:1 allocation occur as per a computerised randomisation schedule stratified by site (to account for variation in rehabilitation programmes between Canada and the USA) using permutated blocks of random sizes and to ensure equal assignment of the MyndMove and the CT at each site. The block sizes will not be disclosed to ensure concealment. Sufficient randomisation sequence allocation, prior to study activation, will be generated to permit the enrolment and drop-out of at least $40 \%$ of the total sample size.

Participants who provide signed informed consent, meet all inclusion/exclusion criteria for the study and complete the baseline visit will be randomly assigned to one of the two treatment arms requested directly from REDCap system. Through REDCap, the randomisation allocation will be provided to the study coordinator. The study coordinator will then provide the information about treatment allocation to the participant and treating therapist. The therapist who is the outcome assessor will be blinded to the treatment allocation. All therapists (whether treating or assessing) will be licenced in physical or occupational therapy.

\section{Intervention}

Participants randomised to the MyndMove therapy group will receive FES therapy bilaterally at the therapist discretion based on clinical presentation/dominance and participant's goals. Treatment will be provided in 1-hour sessions per day for a minimum-maximum of 36-40 sessions delivered no less than three times per week and up to five times per week within a 14-week period of time. Over the course of the sessions, the participants will progress through various movement sequences aimed at regaining natural, unassisted voluntary movements in the affected $\operatorname{limb}(\mathrm{s})$. The proposed volume of therapy is guided by discussions with clinicians experienced with delivery of MyndMove therapy along with previous clinical research studies. ${ }^{27}$

The type and frequency of protocols used will follow a standardised regimen in order to minimise cointervention variation across sites. ${ }^{27}$ Training for MyndMove will be provided prior to the initiation of the study. Guidance regarding protocol selection, sequence and frequency of repetition will be provided as a part of the training by MyndTec. The selection of protocols used during each treatment session will be captured. During each treatment session, therapists will select from a menu of preprogrammed stimulation protocols to facilitate various task-specific movements (table 1). Movement practice may be massed or distributed, depending on the tolerance of the participant (ie, muscle fatigue).

The CT intervention serves as an active control group and will use conventional rehabilitative therapy with control for the schedule, form and intensity of
Table 1 Example MyndMove protocols

Muscles stimulated with

Movement practised* MyndMove†

\begin{tabular}{|c|c|}
\hline Palmar grasp $\ddagger$ & $\begin{array}{l}\text { Flexor digitorum superficialis } \\
\text { and profundus. } \\
\text { Thenar muscles. } \\
\text { Extensor digitorum. }\end{array}$ \\
\hline Lateral pinch grasp $\ddagger$ & $\begin{array}{l}\text { Flexor digitorum superficialis } \\
\text { and profundus. } \\
\text { Thenar muscles. } \\
\text { Extensor digitorum. }\end{array}$ \\
\hline Pinch grasp $\ddagger$ & $\begin{array}{l}\text { Thenar muscles. } \\
\text { Extensor digitorum. } \\
\text { First lumbrical. }\end{array}$ \\
\hline Lumbrical grasp & $\begin{array}{l}\text { Thenar muscles. } \\
\text { Extensor digitorum. } \\
\text { First, second and third } \\
\text { lumbricals. }\end{array}$ \\
\hline Tripod grasp & $\begin{array}{l}\text { Flexor digitorum superficialis. } \\
\text { Thenar muscles. } \\
\text { Extensor digitorum. } \\
\text { Second dorsal interosseous. }\end{array}$ \\
\hline $\begin{array}{l}\text { Side reach with finger } \\
\text { extension }\end{array}$ & $\begin{array}{l}\text { Biceps. } \\
\text { Triceps. } \\
\text { Middle deltoid. } \\
\text { Extensor digitorum. } \\
\text { Extensor carpi radialis longus. } \\
\text { Extensor carpi ulnaris. }\end{array}$ \\
\hline $\begin{array}{l}\text { Forward reach and } \\
\text { grasp }\end{array}$ & $\begin{array}{l}\text { Biceps. } \\
\text { Triceps. } \\
\text { Posterior deltoid. } \\
\text { Anterior deltoid. } \\
\text { Extensor digitorum. } \\
\text { Extensor carpi radialis longus. } \\
\text { Extensor carpi ulnaris. } \\
\text { Flexor digitorum superficialis } \\
\text { and profundus. } \\
\text { Thenar muscles. }\end{array}$ \\
\hline Hand to mouth & $\begin{array}{l}\text { Biceps. } \\
\text { Triceps. } \\
\text { Anterior deltoid. }\end{array}$ \\
\hline
\end{tabular}

*The movement is demonstrated for the participant by the therapist. The therapist then instructs the participant to voluntarily attempt the movement for about $10 \mathrm{~s}$, after which electrical stimulation is provided with the appropriate MyndMove protocol. ${ }^{39}$ †Muscles not listed in order of stimulation.

fUnilateral or bilateral stimulation may be used.

participant-therapist interactions and therapeutic activities in the MyndMove therapy group. During each treatment session, participants will receive CT of equivalent duration to the 1-hour sessions per day of MyndMove therapy. The type and frequency of interventions used will follow a standardised regimen developed by consensus across the centres for the CT in order to minimise intervention variation across sites. Conventional upper limb rehabilitation therapy, at the local institution, may include any or all of the following: (A) facilitation of reaching or 
prehension movements; (B) bilateral task-specific movement practice (distributed or massed, dependent on participant tolerance); (C) range of motion and mobilisation of joints; (D) splinting; (E sensorimotor stimulation (eg, TENS, acupuncture, muscle stimulation and biofeedback); (F) electrical stimulation (for strength, not function); and (G) reduction of oedema, if needed. The use of other FES devices during the course of the study will not be permitted. The Tempate for Intervention Description and Replication (TiDieR) checklist will be used to report results.

All other rehabilitation services will be provided throughout the intervention and follow-up period. This concomitant care, which may influence outcomes, will be captured throughout the study by self-report through the use of a healthcare resource utilisation questionnaire, provided to the participant and confirmed by the study staff. During the intervention period, the questionnaire will be completed by the participant to record any rehabilitation services and provide a categorical description of the treatment provided and duration of treatment sessions. This information will be reviewed by study staff and verified with the participant.

For all treatment arms, adherence to therapy will be captured to document any missed research therapy visits. This will allow for the assessment of the effectiveness of the treatment and the practicality of daily administration of the treatment. A per-protocol analysis will be completed using only data from those participants completing at least 30 treatments, which corresponds to $75 \%$ of allocated treatments.

\section{Data collection and management}

The Biostatistics Unit will provide data management and analysis for the study. All data will be deidentified to maintain confidentiality and captured on paper case report forms. Key data will be entered at each site directly into the electronic database created in REDCap.

An independent research monitor will be appointed, with expertise consonant with the nature of risk(s) identified within the research protocol. The duties, authorities and responsibilities of the independent research monitor will include: observation of recruitment and enrolment procedures and the consent process for individuals, overseeing study interventions and interactions, reviewing monitoring plans and unanticipated problems involving risk to subjects or others reports; and overseeing data matching, data collection and analysis. Monitoring activities will be performed both on-site and off-site according to Good Clinical Practices (GCP) guidelines. A MyndTec Study Monitor will conduct the site initiation visit, periodic site visits (with the independent research monitor) and a close-out visit for each site.

\section{Schedule of data collection}

A schedule of assessments and data collection is provided in table 2.

\section{Adverse events (AEs) and serious adverse events (SAEs)}

All AEs will be recorded and used to assess participant safety. AE will be recorded on the appropriate case report forms from the time written informed consent is obtained until completion of the study or until resolution of the reportable event. Information to be collected includes the description of the AE, date and time of onset, severity, duration, causality, outcome and relationship to the study procedure.

An $\mathrm{AE}$ or suspected $\mathrm{AE}$ is considered 'serious' if, in the view of either the investigator or sponsor, it results in any of the following outcomes: (1) leads to death, (2) is life threatening, or places the participant at immediate risk of death, (3) requires or prolongs inpatient hospitalisation, (4) results in a significant, persistent or permanent change, impairment, damage or disruption in the participant's body function/structure, physical activities and/ or quality of life, (5) results in congenital anomaly/birth defect or (6) any other serious or important event that may jeopardise the participant and may require medical or surgical intervention (treatment) to prevent one of the other outcomes.

All AEs and SAEs will be followed until: (1) AE is resolved, (2) AE is declared clinically insignificant, (3) AE has stabilised, (4) participant is lost to follow-up or withdraws consent, (5) participant completes study, including required follow-up visits or (6) study closure.

MyndTec Inc shall reimburse all reasonable and necessary expenses incurred for medical care received by study participants, including hospitalisation, in the treatment of any injury or illness sustained by a clinical trial participant as a result of receiving treatment with MyndMove therapy in the study.

\section{Outcomes}

\section{Primary outcome}

The primary outcome for the study is the change in SCIM-SC between baseline and end of treatment (14 weeks). This is the basis for the a priori sample size and sensitivity estimates. The SCIM is a disability scale that has been specifically developed to evaluate the functional outcomes of people with traumatic and non-traumatic SCI. ${ }^{28}$

\section{Secondary outcomes}

Additional secondary analyses of the SCIM self-care and mobility subscales will be performed at the interim, endof-treatment assessment and end-of-study assessments (see table 2 schedule of assessments). The GRASSP test ${ }^{29-31}$ is a multimodality test designed to assess the integration of sensorimotor hand and upper limb impairment and function. The baseline scores for each of the GRASSP subscales will be compared with the scores at interim, endof-treatment assessment and end-of-study assessment. The TRI-HFT ${ }^{32}$ was developed to evaluate improvements in the gross motor function of the unilateral grasp due to FES for reaching and grasping treatment. The baseline scores for each of the TRI-HFT subscales will be compared with 
Table 2 A summary of assessments and data collection

the scores at the end-of-treatment assessment and end-ofstudy assessments. The SCI-QOL measurement system is a multifaceted system of measuring participants reported outcomes across a wide variety of functioning specifically targeted for individuals with SCI. ${ }^{33}$ Participants will complete 9 out of 22 areas of measure in the SCI-QOL (table 3). The baseline scores for each of the SCI-QOL subscales will be compared with the scores at the endof-treatment assessment and end-of-study assessment. A healthcare resource utilisation questionnaire to capture inpatient, outpatient and community-based rehabilitation and healthcare services during the follow-up period will also be collected. The total number of minutes used from baseline to the end-of-study assessment will be compared between groups. Participants will be asked to complete an end-of-therapy questionnaire that consists of three openended questions to understand their acceptance and
Treatment period

Early termination assessment

$\begin{array}{ll}\text { Interim } & \begin{array}{l}\text { End-of-treatment } \\ \text { assessment (after }\end{array} \\ \text { assessment } & \text { 40th treatment } \\ \text { (after 20th } & \text { session/14 weeks } \\ \text { treatment } & \begin{array}{l}\text { post first treatment } \\ \text { session) }\end{array} \\ \text { visit) }\end{array}$

End-of-study follow-up assessment $(24$ weeks post first treatment visit)

\begin{tabular}{|c|c|c|c|c|c|c|}
\hline Events & visit & visit & Randomisation & session) & visit) & treatment visit) \\
\hline \multicolumn{7}{|l|}{ Consent } \\
\hline Informed consent form & $\times$ & & & & & \\
\hline \multicolumn{7}{|l|}{ Eligibility } \\
\hline Inclusion/exclusion criteria & $x$ & & & & & \\
\hline Enrolment & & & $x$ & & & \\
\hline \multicolumn{7}{|l|}{ Interventions } \\
\hline \multicolumn{7}{|l|}{ Intensive conventional therapy } \\
\hline \multicolumn{7}{|l|}{ Assessments } \\
\hline Demographics and social status & & $x$ & & & & \\
\hline General health history & & $x$ & & & & \\
\hline SCIM & $x$ & $x$ & & $x$ & $x$ & $x$ \\
\hline GRASSP & & $x$ & & $x$ & $x$ & $x$ \\
\hline TRI-HFT & & $x$ & & & $x$ & $x$ \\
\hline \multicolumn{7}{|l|}{ Participation and quality of life } \\
\hline AE/SAE & & & & $x$ & $x$ & $x$ \\
\hline SCI-QOL & & $x$ & & & $x$ & $x$ \\
\hline $\begin{array}{l}\text { Healthcare resource utilisation } \\
\text { questionnaire }\end{array}$ & & $x$ & & $x$ & $x$ & $x$ \\
\hline End of Therapy Questionnaire & & & & & $x$ & \\
\hline
\end{tabular}

impression of the therapy they received in the trial. See table 3 for the analysis plan for each secondary outcome.

\section{Participant and disease characteristics (demographics and} SCI info)

The following participant characteristics will be captured: AIS grade and neurological level, concomitant medications, biological sex, age, race, ethnicity, marital status, number of members in household, years of education, primary occupation, family income range, handedness, international SCI upper extremity basic data set, general medical history, cause of SCI, current medical complications related to SCI, surgical history, current medical symptoms, smoking status and alcohol consumption.

\section{Data analysis plan}

The analysis and reporting of the results with follow the Consolidated Standards of Reporting Trials guideline ( 
Table 3 Summary of the analysis for each objective, outcome and corresponding hypothesis

\begin{tabular}{|c|c|c|c|}
\hline Variable/outcome & Hypothesis & Outcome measure (type of outcome) & $\begin{array}{l}\text { Methods of } \\
\text { analysis }\end{array}$ \\
\hline $\begin{array}{l}\text { 1. Primary } \\
\text { Upper extremity } \\
\text { function. }\end{array}$ & $\begin{array}{l}\text { FES intervention (I) is better than } \\
\text { conventional therapy control (C). }\end{array}$ & SCIM-SC subscale score. & ANCOVA. \\
\hline $\begin{array}{l}\text { 2. Secondary } \\
\text { Limb function. }\end{array}$ & $\mathrm{I}$ is better than $\mathrm{C}$. & SCIM mobility subscale score. & ANCOVA. \\
\hline Upper limb function. & $\mathrm{I}$ is better than $\mathrm{C}$. & $\begin{array}{l}\text { GRASSP subscales: } \\
\text { Strength total score. } \\
\text { Sensibility total score. } \\
\text { Qualitative prehension total score. } \\
\text { Quantitative prehension total score. }\end{array}$ & ANCOVA. \\
\hline - Upper limb function. & I is better than $\mathrm{C}$. & $\begin{array}{l}\text { TRI-HFT subscales: } \\
\text { Object manipulation score. } \\
\text { Wooden block score. } \\
\text { Cylinder torque. } \\
\text { Credit card force. } \\
\text { Wooden bar displacement length. }\end{array}$ & ANCOVA. \\
\hline
\end{tabular}

\begin{tabular}{|c|c|c|c|}
\hline - QoL. & I is better than $\mathrm{C}$. & $\begin{array}{l}\text { SCI-QOL subscales: } \\
\text { Basic mobility score. } \\
\text { Fine motor score. } \\
\text { Manual wheelchair score. } \\
\text { Power wheelchair score. } \\
\text { Self-care score. } \\
\text { Independence score. } \\
\text { Pain behaviour score. } \\
\text { Pain interference score. } \\
\text { Satisfaction with social roles and activities score. }\end{array}$ & ANCOVA. \\
\hline - Safety. & I is better than $\mathrm{C}$. & $\begin{array}{l}\text { Serious and non-serious adverse events, total number of } \\
\text { each per group. }\end{array}$ & Descriptive. \\
\hline $\begin{array}{l}\text { Healthcare resource } \\
\text { utilisation. }\end{array}$ & $\begin{array}{l}\text { Reduced healthcare resource } \\
\text { utilisation with I compared with C. }\end{array}$ & $\begin{array}{l}\text { Healthcare resource utilisation questionnaire, total number } \\
\text { of minutes. }\end{array}$ & ANCOVA. \\
\hline $\begin{array}{l}\text { 3. Sensitivity analyses: } \\
\text { Per-protocol. } \\
\text { Missing data based } \\
\text { imputed based on } \\
\text { LOCF. } \\
\text { Adjusted analysis } \\
\text { with key baseline } \\
\text { characteristics: age, } \\
\text { baseline function and } \\
\text { QoL. }\end{array}$ & $\begin{array}{l}\text { Results of analysis of primary } \\
\text { analysis will remain robust. }\end{array}$ & SCIM-SC score. & $\begin{array}{l}\text { ANCOVA with } \\
\text { multivariable } \\
\text { analysis for } \\
\text { adjusted } \\
\text { analysis. }\end{array}$ \\
\hline \multicolumn{4}{|c|}{$\begin{array}{l}\text { Important remarks: } \\
\text { In all analyses, results will be expressed as coefficient, SEs, corresponding } 95 \% \text { and associated p values. } \\
\text { Goodness-of-fit will be assessed by examining the residuals for model assumptions and } \chi^{2} \text { test of goodness-of-fit. }\end{array}$} \\
\hline
\end{tabular}

ANCOVA, analysis of covariance; C, control; FES, functional electrical stimulation; GRASSP, Graded Redefined Assessment of Strength, Sensibility and Prehension; I, intervention; LOCF, last observation carried forward; QOL, quality of life; SCIM, Spinal Cord Independence Measure III; SCIMSC, Spinal Cord Independence Measure III self-care sub-scale; SCI-QOL, Spinal Cord Injury-Quality of Life; TRI-HFT, Toronto Rehab Institute Hand Function Test.

www.consort-statement.org). The statistician/data analyst will be blinded to the study group. The process of participant selection and flow throughout the study will be summarised using a flow diagram. The analysis results of participant demographics and baseline outcome variables (both primary and secondary) will be summarised using descriptive summary measures: expressed as mean (SD) or median (IQR) for continuous variables and number (per cent) for categorical variables. We will adopt an intentionto-treat principle to analyse all outcomes. We will also use multiple imputation to handle missing outcome data. ${ }^{34}$
Research has shown that this is the most optimal strategy for handling missing outcome data in trials under the assumption of missing at random. ${ }^{35}$ All statistical tests will be performed using two-sided tests at the 0.05 level of significance. The overall level of significance will not be adjusted for multiple testing for secondary outcomes because these are exploratory. We will use ANCOVA for the analyses of both primary and secondary outcomes, with treatment group as an independent variable and baseline values of each outcome as a covariate. For all models, the results will be expressed as mean difference, 
corresponding two-sided 95\% CIs and associated $\mathrm{p}$ values. $\mathrm{P}$ values will be reported to three decimal places with values less than 0.001 reported as $<0.001$. We will conduct some sensitivity analyses to assess the robustness of the results: (1) per-protocol analysis: this analysis will be based only on participants with complete data that completed study procedures as per-protocol; (2) using last observation carried forward (LOCF) for missing data: this analysis will use the LOCF to impute missing data; (3) adjusted analysis: this analysis will adjust for some baseline variables that we think may impact the results if not balanced. These include age, time postinjury, baseline function, baseline quality of life and, potentially, site. To the extent that these sensitivity analyses yield similar results to the main analysis, inferences about the primary outcome will be strengthened ${ }^{36}{ }^{37}$ Goodness-of-fit will be assessed by examining the residuals for model assumptions and $\chi^{2}$ test of goodness-of-fit. Please see table 3 for a summary of the analysis for each objective, outcome and corresponding hypothesis. All analyses will be performed using SAS V.9.2.

\section{Patient and public involvement}

Patients were not involved in the design of this protocol. Collaborations will be developed with SCI community organisations to codevelop lay descriptions of the results of the trial for the public.

\section{ETHICS AND DISSEMINATION}

The study design is described according to the Standard Protocol Items: Recommendations for Interventional Trials reporting guidelines. ${ }^{38}$ This study has ethics approval from: MetroHealth System Institutional Review Board (IRB18-0751); University Health Network Research Ethics Board (REB17-6029); University of Texas Health Science Center IRB (HSCMS-18-0862); Advarra IRB for HealthTech Connex Centre for Neurology Studies (Pro00030094); as well as approval from the US Army Medical Research and Materiel Command, Office of Research Protections and Human Research Protection Office. Any changes to the protocol will not be implemented until ethics approvals have been obtained. Amendments will be numbered in a sequential manner and assigned an amendment date and version.

Data collected as a part of the study will be maintained at Biostatistics Unit on behalf of the investigators. The initial evaluation of the clinical study results will be provided to the investigators and to MyndTec Inc. MyndTec Inc will not prevent publication of the results regardless of the outcome of the study. Dissemination of the results of the study will be made at peer-reviewed academic meetings and through peer-reviewed medical journals. Participant confidentiality will be maintained in all analyses and presentations.

\section{Author affiliations}

${ }^{1}$ Department of Physical Medicine and Rehabilitation, MetroHealth System, Cleveland, Ohio, USA

${ }^{2}$ Department of Physical Medicine and Rehabilitation, Case Western Reserve University, Cleveland, Ohio, USA

${ }^{3}$ Department of Physical Medicine and Rehabilitation, University of Texas Health Science Center at Houston, Houston, Texas, USA

${ }^{4}$ The Institute for Rehabilitation and Research (TIRR), Houston, Texas, USA ${ }^{5}$ Centre for Neurology Studies, Health Tech Connex, Surrey, British Columbia, Canada

${ }^{6}$ Department of Health Research Methods, Evidence, and Impact, McMaster University Faculty of Health Sciences, Hamilton, Ontario, Canada

${ }^{7}$ Toronto Health Economics and Technology Assessment (THETA) Collaborative, University of Toronto, Toronto, Ontario, Canada

${ }^{8}$ TELUS Health, Toronto, Ontario, Canada

${ }^{9}$ Toronto Rehabilitation Institute, University Health Network, Toronto, Ontario, Canada ${ }^{10}$ Department of Physical Therapy, University of Toronto, Toronto, Ontario, Canada

${ }^{11}$ The KITE Research Institute, University Health Network, Toronto, Ontario, Canada

${ }^{12}$ Institute of Biomaterials and Biomedical Engineering, University of Toronto, Toronto, Ontario, Canada

${ }^{13}$ Biostatistics Unit, St. Joseph's Healthcare, Hamilton, Ontario, Canada

Contributors All of the authors made substantial contributions to study design; KDA, JRW, RK, JP, LT and KEM are involved in conduct; all authors were involved in drafting or revising this protocol manuscript for important intellectual content and gave final approval of the version to be published; KDA, JRW, RK, JP, MRP, LT and KEM will be involved in the analyses and reporting of results.

Funding This trial is funded in part under the USAMRMC, award number W81XWH16-1-0790, SC150251. MyndTec Inc. is responsible for training and distribution related to the MyndMove device and resolution of clinical and technical devicerelated issues throughout the study. MyndTec Inc provided additional funding for costs which were not covered by funding. In addition, funding provided through the grant will be administered to the participating sites through MyndTec Inc.

Competing interests MyndTec Inc is the contracting organisation of this study and through funding provided by US Army Medical Research and Materiel Command, researchers are reimbursed for doing this study. All investigators have an interest in completing the study.

Patient consent for publication Not required.

Provenance and peer review Not commissioned; externally peer reviewed.

Open access This is an open access article distributed in accordance with the Creative Commons Attribution Non Commercial (CC BY-NC 4.0) license, which permits others to distribute, remix, adapt, build upon this work non-commercially, and license their derivative works on different terms, provided the original work is properly cited, appropriate credit is given, any changes made indicated, and the use is non-commercial. See: http://creativecommons.org/licenses/by-nc/4.0/.

\section{ORCID iDs}

Kim D Anderson http://orcid.org/0000-0001-9252-161X

James M Bowen http://orcid.org/0000-0002-6457-2337

\section{REFERENCES}

1 Anderson KD. Targeting recovery: priorities of the spinal cord-injured population. J Neurotrauma 2004;21:1371-83.

2 Noonan VK, Fingas M, Farry A, et al. Incidence and prevalence of spinal cord injury in Canada: a national perspective. Neuroepidemiology 2012;38:219-26.

3 Hoshimiya N, Handa Y. A master-slave type multi-channel functional electrical stimulation (FeS) system for the control of the paralyzed upper extremities. Automedica 1989;11:209-20.

4 Handa $\mathrm{Y}$, Handa T, Ichie M, et al. Functional electrical stimulation (FES) systems for restoration of motor function of paralyzed muscles--versatile systems and a portable system. Front Med Biol Eng 1992;4:241-55.

5 Nathan RH, Ohry A. Upper limb functions regained in quadriplegia: a hybrid computerized neuromuscular stimulation system. Arch Phys Med Rehabil 1990;71:415-21.

6 IJzerman MJS, Stoffers TS, et al. The NESS Handmaster orthosis: restoration of hand function in $\mathrm{C} 5$ and stroke patients by means of electrical stimulation. J Rehabil Sci 1996;9:86-9. 
7 Snoek GJ, IJzerman MJ, in 't Groen FA, et al. Use of the NESS handmaster to restore handfunction in tetraplegia: clinical experiences in ten patients. Spinal Cord 2000;38:244-9.

8 Prochazka A, Gauthier M, Wieler M, et al. The bionic glove: an electrical stimulator garment that provides controlled GRASP and hand opening in quadriplegia. Arch Phys Med Rehabil 1997;78:608-14.

9 Popović D, Stojanović A, Pjanović A, et al. Clinical evaluation of the bionic glove. Arch Phys Med Rehabil 1999;80:299-304.

10 Popovic MR, Popovic DB, Keller T. Neuroprostheses for grasping. Neurol Res 2002;24:443-52.

11 Popovic MR, Thrasher TA, Zivanovic V, et al. Neuroprosthesis for retraining reaching and grasping functions in severe hemiplegic patients. Neuromodulation 2005;8:58-72.

12 Popovic MR, Thrasher TA, Adams ME, et al. Functional electrical therapy: retraining grasping in spinal cord injury. Spinal Cord 2006;44:143-51.

13 Rebersek S, Vodovnik L. Proportionally controlled functional electrical stimulation of hand. Arch Phys Med Rehabil 1973;54:378-82.

14 Peckham PH, Marsolais EB, Mortimer JT. Restoration of key grip and release in the $\mathrm{C} 6$ tetraplegic patient through functional electrical stimulation. J Hand Surg Am 1980;5:462-9.

15 Peckham PH, Mortimer JT, Marsolais EB. Controlled prehension and release in the $\mathrm{C} 5$ quadriplegic elicited by functional electrical stimulation of the paralyzed forearm musculature. Ann Biomed Eng 1980;8:369-88.

16 Kilgore KL, Peckham PH, Thrope GB, et al. Synthesis of hand GRASP using functional neuromuscular stimulation. IEEE Trans Biomed Eng 1989;36:761-70.

17 Wuolle KS, Van Doren CL, Thrope GB, et al. Development of a quantitative hand GRASP and release test for patients with tetraplegia using a hand neuroprosthesis. J Hand Surg Am 1994:19:209-18.

18 Mangold S, Keller T, Curt A, et al. Transcutaneous functional electrical stimulation for grasping in subjects with cervical spinal cord injury. Spinal Cord 2005;43:1-13.

19 Beekhuizen KS, Field-Fote EC. Sensory stimulation augments the effects of massed practice training in persons with tetraplegia. Arch Phys Med Rehabil 2008;89:602-8.

20 Kowalczewski J, Chong SL, Galea M, et al. In-Home telerehabilitation improves tetraplegic hand function. Neurorehabil Neural Repair 2011;25:412-22.

21 Teferra M. Functional electrical stimulation (FeS): review. IJLRET 2017;3:95-101.

22 Popovic MR, Kapadia N, Zivanovic V, et al. Functional electrical stimulation therapy of voluntary grasping versus only conventiona rehabilitation for patients with subacute incomplete tetraplegia: a randomized clinical trial. Neurorehabil Neural Repair 2011;25:433-42.

23 Nagai MK, Marquez-Chin C, Popovic MR. Why is functional electrical stimulation therapy capable of restoring motor function following severe injury to the central nervous system? In: Tuszynski $\mathrm{MH}$, ed. Translational neuroscience. New York, NY: Springer Science and Business Media, 2016: 479-98.
24 Kapadia N, Zivanovic V, Popovic MR. Restoring voluntary grasping function in individuals with incomplete chronic spinal cord injury: pilot study. Top Spinal Cord Inj Rehabil 2013;19:279-87.

25 Scivoletto G, Tamburella F, Laurenza L, et al. The spinal cord independence measure: how much change is clinically significant for spinal cord injury subjects. Disabil Rehabil 2013;35:1808-13.

26 Scivoletto G, Tamburella F, Laurenza L, et al. Distribution-based estimates of clinically significant changes in the International standards for neurological classification of spinal cord injury motor and sensory scores. Eur J Phys Rehabil Med 2013;49:373-84.

27 Hebert DA, Bowen JM, Ho C, et al. Examining a new functional electrical stimulation therapy with people with severe upper extremity hemiparesis and chronic stroke: a feasibility study. Br J Occup Ther 2017;80:651-9.

28 Catz A, Itzkovich M, Agranov E, et al. SCIM-spinal cord independence measure: a new disability scale for patients with spinal cord lesions. Spinal Cord 1997;35:850-6.

29 Kalsi-Ryan S, Beaton D, Mcllroy W, et al. Quantification of multimodality sensation of the hand in cervical spinal cord injury. J Spinal Cord Med 2006;29:311.

30 Kalsi-Ryan S, Beaton D, Curt A, et al. The graded redefined assessment of strength sensibility and prehension: reliability and validity. J Neurotrauma 2012;29:905-14.

31 Kalsi-Ryan S, Curt A, Verrier MC, et al. Development of the graded redefined assessment of strength, sensibility and prehension (GRASSP): reviewing measurement specific to the upper limb in tetraplegia. J Neurosurg Spine 2012;17:65-76.

32 Kapadia N, Zivanovic V, Verrier M, et al. Toronto rehabilitation institutehand function test: assessment of gross motor function in individuals with spinal cord injury. Top Spinal Cord Inj Rehabil 2012;18:167-86.

33 Tulsky DS, Kisala PA, Victorson D, et al. Overview of the Spinal Cord Injury--Quality of Life (SCI-QOL) measurement system. J Spinal Cord Med 2015;38:257-69.

34 Little RJA, Rubin DB. Statistical analysis with missing data. New York, NY: John Wiley \& Sons, 1987.

35 Zhang Y, Alyass A, Vanniyasingam T, et al. A systematic survey of the methods literature on the reporting quality and optimal methods of handling participants with missing outcome data for continuous outcomes in randomized controlled trials. J Clin Epidemiol 2017:88:67-80.

36 Thabane L, Mbuagbaw L, Zhang S, et al. A tutorial on sensitivity analyses in clinical trials: the what, why, when and how. BMC Med Res Methodol 2013;13:92.

37 de Souza RJ, Eisen RB, Perera S, et al. Best (but oft-forgotten) practices: sensitivity analyses in randomized controlled trials. Am J Clin Nutr 2016;103:5-17.

38 Chan A-W, Tetzlaff JM, Altman DG, et al. Spirit 2013 statement: defining standard protocol items for clinical trials. Ann Intern Med 2013;158:200-7

39 Kapadia N, Moineau B, Popovic MR. Functional electrical stimulation therapy for retraining reaching and grasping after spinal cord injury and stroke. Front Neurosci 2020;14:718. 